INPLASY

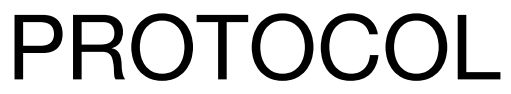

To cite: Wang et al.

Acupuncture response rates in chronic constipation: A protocol for the systematic review and meta-analysis. Inplasy protocol 202210041. doi:

10.37766/inplasy2022.1.0041

Received: 09 January 2022

Published: 09 January 2022

Corresponding author:

Lu Wang

wanglu@stu.cdutcm.edu.cn

Author Affiliation:

Chengdu university of TCM

Support: National Natural

Science Found.

Review Stage at time of this submission: Preliminary searches.

Conflicts of interest:

None declared.

\section{Acupuncture response rates in chronic constipation: A protocol for the systematic review and meta-analysis}

\author{
Wang, L1; Xi, MH2; Qin, HY3; Cao, W4; Chen, Y5; Zheng, QH6;
} Zou, ZH77 Xiao, XJ8; Shi, YZ79; Su, CG'10; Hou, YJ11; Li, Y12.

Review question / Objective: Some high-quality randomized controlled trials and meta-analysis have demonstrated the effectiveness and safety of acupuncture. However, the factors that related to the therapeutic effect of acupuncture need to be further explored.

Condition being studied: Several randomized controlled trials and meta-analyses have demonstrated the efficacy and safety of acupuncture. However, as a part of traditional Chinese medicine, there are many factors that affect the efficacy of acupuncture, which is worthy of further research and exploration.

Information sources: PubMed, Cochrane Library, Web of Science and Embase were electronically searched from their inception to December 2021.

INPLASY registration number: This protocol was registered with the International Platform of Registered Systematic Review and Meta-Analysis Protocols (INPLASY) on 09 January 2022 and was last updated on 09 January 2022 (registration number INPLASY202210041).

\section{INTRODUCTION}

Review question / Objective: Some highquality randomized controlled trials and meta-analysis have demonstrated the effectiveness and safety of acupuncture. However, the factors that related to the therapeutic effect of acupuncture need to be further explored.

Condition being studied: Several randomized controlled trials and metaanalyses have demonstrated the efficacy and safety of acupuncture. However, as a part of traditional Chinese medicine, there 
are many factors that affect the efficacy of acupuncture, which is worthy of further research and exploration.

\section{METHODS}

Participant or population: Patients with chronic constipation, functional constipation or irritable bowel syndrome with predominant constipation.

Intervention: All types of acupuncture intervention methods.

Comparator: There is no clear restriction on the comparison of the study.

Study designs to be included: Randomized controlled trials.

Eligibility criteria: The Rome IV/III/II criteria or other related guidelines.

Information sources: PubMed, Cochrane Library, Web of Science and Embase were electronically searched from their inception to December 2021.

Main outcome(s): The primary outcome assessed is the magnitude of the acupuncture response rate.

Quality assessment / Risk of bias analysis: The Cochrane Collaborative Bias Risk Tool will be used to evaluate and describe the six sources of bias in each RCT, namely random sequence generation, allocation concealment, blinding of participants and personnel, blinding of outcome assessment, incomplete outcome data, selective reporting and other bias.

Strategy of data synthesis: R software will be used for performing all the statistical analyses, such as calculating the pooled response rate as well as its $95 \%$ confidence interval and drawing the forest plot.

Subgroup analysis: Subgroup analyses identified the possible factors that contributed to the heterogeneity, such as different acupuncture stimulation parameters, different control groups, participants' age or disease course.

Sensitivity analysis: Sensitivity analysis will be conducted to test the stability of the results.

Country(ies) involved: China.

Keywords: acupuncture, chronic constipation, response rate.

Contributions of each author:

Author 1 - Lu Wang.

Author 2 - Meng-han X.

Author 3 - Hai-yan Qin.

Author 4 - Wei Cao.

Author 5 - Ying Chen.

Author 6 - Qian-hua Zheng.

Author 7 - Zi-hao Zou.

Author 8 - Xian-jun Xiao.

Author 9 - Yun-zhou Shi.

Author 10 - Cheng-guo Su.

Author 11 - Yu-jun Hou.

Author 12 - Ying Li. 